\title{
Original
}

Journal of Hard Tissue Biology 23[4] (2014) 389-398

C 2014 The Hard Tissue Biology Network Association Printed in Japan, All rights reserved. CODEN-JHTBFF, ISSN 1341-7649

\section{Osteogenic Effect of Local Administration of Fluvastatin using a Fluvastatin- gelatin Complex in Senile Osteoporosis Model Rats}

\author{
Hiromitsu Yasuda ${ }^{1,2)}$, Koji Tanabe ${ }^{2,3)}$, Toru Sato ${ }^{1)}$, Shuntaro Nomoto ${ }^{1)}$, Tadashi Miura ${ }^{2)}$ and Masao Yoshinari ${ }^{2)}$ \\ 1) Department of Crown and Bridge Prosthodontics, Tokyo Dental College, Tokyo, Japan \\ 2) Division of Oral Implants Research, Oral Health Science Center, Tokyo Dental College, Tokyo, Japan \\ 3) Department of Pharmacology, Tokyo Dental College, Tokyo, Japan \\ (Accepted for publication, July 1, 2014)
}

\begin{abstract}
Statins, inhibitors of 3-hydroxy-2-methyl-glutaryl coenzyme A reductase, were recently reported to promote the differentiation of osteoblasts produced by the stimulation of bone morphogenetic protein-2 (BMP2). The aim of this study was to investigate the osteogenic effect of a local administration of fluvastatin in senile osteoporosis model rats using a fluvastatin-gelatin complex. The gelatin-hydrogel was put into cylindrical titanium tubes $1.5 \mathrm{~mm}$ in diameter and $2.2 \mathrm{~mm}$ high. It was then crosslinked by ultraviolet irradiation, after which it was lyophilized. Subsequently, the carriers were placed in the fluvastatin solution $(300 \mu \mathrm{M})$ for one day to bind the alkaline gelatin and acidic statin electrostatically (fluvastatin-gelatin complex; statin group). A control group consisted of the carriers immersed in sterilized water. Specimens of both the statin group and the control group were implanted into the femur of 15-week-old male senile osteoporosis model rats (SHRSP). Radiographic analysis, histologic examination, and immunohistochemical staining (BMP-2 and Runx2) were then performed. At 14 and 21 days, the bone volumes of the newly formed bone in the statin group was significantly higher compared to those of the control group $(P<0.05)$. Histological observation showed that a high level of new bone formation was present in the statin group compared to that of the control group during all time periods. At 7 days, positive immune reactions with BMP-2 and Runx2 were seen in the statin group. The local administration of the fluvastatin-gelatin complex facilitated bone formation in senile osteoporosis model rats.
\end{abstract}

Key words: Fluvastatin-gelatin complex, Senile osteoporosis, Local-administration, Bone formation, Micro$\mathrm{CT}$

\section{Introduction}

Osteoporosis is a common systemic disease that is related to risk factors for compromised osseointegration. ${ }^{1)}$ A decrease in bone mass causes a reduction in the contact area between implant and bone, and may also cause a reduction in support of the implant because of thinning of the surrounding bone tissue ${ }^{2}$. Several osteogenic drugs have been used in patients with osteoporosis.

Statins, or 3-hydroxy-3- methylglutaryl coenzyme A (HMGCoA) reductase inhibitors, were introduced early in the 1990s and have been widely used for hyperlipidemic patients ${ }^{3,4}$. Since locally applied statins were found to be potent stimulators of bone formation $^{5}$, the possibility of using these compounds as practical bone anabolic agents has been postulated. Song et al. reported that statins promote osteoblast differentiation and expression of BMPs in mouse bone marrow stromal cells ${ }^{6}$.

Many studies of bone formation in vivo using statins reported the effect of systemic administration ${ }^{7-13)}$. Some authors reported

Correspondence to: Dr. Masao Yoshinari, Division of Oral Implants Research, Oral Health Science Center, Tokyo Dental College, 2-9-18 Misaki-cho, Chiyoda-ku, Tokyo, Japan; Tel.: +81-3-6380-9266; Fax: +813-6380-9606; E-mail: yosinari@tdc.ac.jp the osteogenic effects of the systemic administration of statins on osteoporosis using ovariectomized rats ${ }^{14-17}$.

However, all statins have high first-pass extraction by the liver; $95 \%$ of statins are metabolized to inactive metabolites and leave a relatively small amount to be absorbed into the blood stream and to reach the bone marrow ${ }^{18,19)}$.

Excessive administration of statins increases the risk of liver damage, kidney disease, and rhabdomyolysis ${ }^{20,21)}$. Thus, a study of the osteogenic effect of the local administration of statins was needed.

The purpose of this study was to clarify the effect of the local administration of fluvastatin, a type of statin drug, on bone formation in bone defects of senile osteoporosis model rats.

\section{Materials and Methods}

Titanium foil (99.5\% purity, Nilaco, Tokyo, Japan), $20 \mu \mathrm{m}$ thick, an alkaline gelatin (isoelectric point of 7.0-9.0, Nitta Gelatin, Osaka, Japan), and fluvastatin sodium salt (Toronto Research Chemicals, Ontario, Canada) were used. The fluvastatin sodium salt of $1.29 \mathrm{mg}$ was dissolved in $10 \mathrm{~mL}$ of sterilized water $(300$ $\mu \mathrm{M}, \mathrm{pH}$ of the solution was 6.0 ) according to a previous study ${ }^{22)}$ 

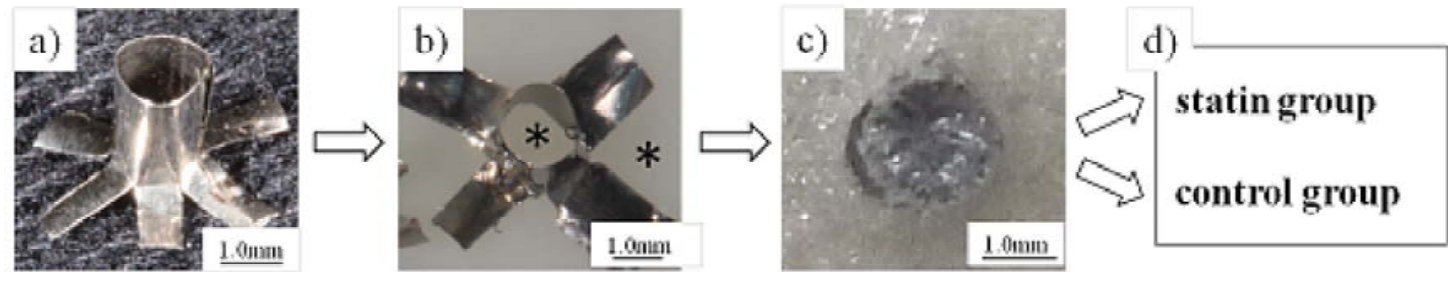

Figure 1. Preparation methods of specimens.

a) Titanium tube, b) Gelatin solution cross-linked with titanium ( * : gelatin solution), c) Lyophilized carrier, d) Specimens (statin and control group) for the animal experiment. Statin group (fluvastatin-gelatin complex): the carriers were immersed in fluvastatin solution. Control group: the carriers were immersed in sterilized water.

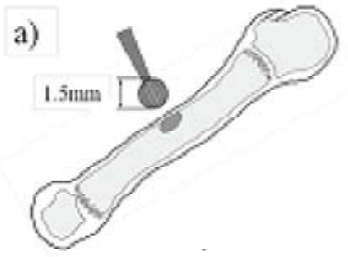

b)

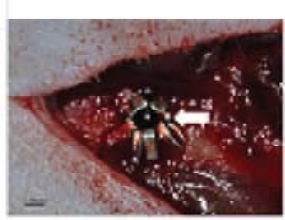

Figure 2. a) Schematic diagram of bone defect model, b) Implantation of the specimen. Arrows indicate specimen.

and a pre-experiment.

\section{Preparation of specimens}

Fig. 1 showed the method used for preparation of the specimens. Cylindrical titanium tubes were prepared to hold the gelatin solution in titanium foil and to specify the region of interest for new bone formation. Titanium tubes were formed from titanium foils (1.5mm diameter $\times 2.2 \mathrm{~mm}$ high) (Fig. 1a). They were then ultrasonically cleaned with $99.5 \%$ ethanol and $99.5 \%$ acetone for $10 \mathrm{~min}$ each after which they were washed with sterilized water.

The titanium tubes were placed in a polystyrene dish filled with alkaline gelatin solution $(2 \mathrm{ml})$ at a concentration of $50 \mathrm{mg} /$ $\mathrm{ml}$. Then, the gelatin solution and the titanium tubes were crosslinked by ultraviolet radiation for $120 \mathrm{~min}$ (Fig. 1b). After crosslinking, the gelatin solution and the titanium tubes were placed in an electric freezer at $-30{ }^{\circ} \mathrm{C}$ for $4 \mathrm{~h}$. Then they were lyophilized for 1 day (Fig. 1c). The lyophilized gelatin solution with the titanium tubes was used as a carrier for the fluvastatin solution and sterilized water.

Two groups of specimens were prepared for the animal experiment. In the statin group (fluvastatin-gelatin complex), the carriers were immersed in fluvastatin solution (300 iM) at $4{ }^{\circ} \mathrm{C}$ for $24 \mathrm{~h}$. In the control group, the carriers were immersed in sterilized water at $4{ }^{\circ} \mathrm{C}$ for $24 \mathrm{~h}$. All experimental procedures were conducted under sterile conditions.

\section{Animal experiment}

15-week-old male spontaneously hypertensive stroke-prone (SHRSP / Izm) rats (body weight 265 \pm 15 g, Sankyo Labo Service,

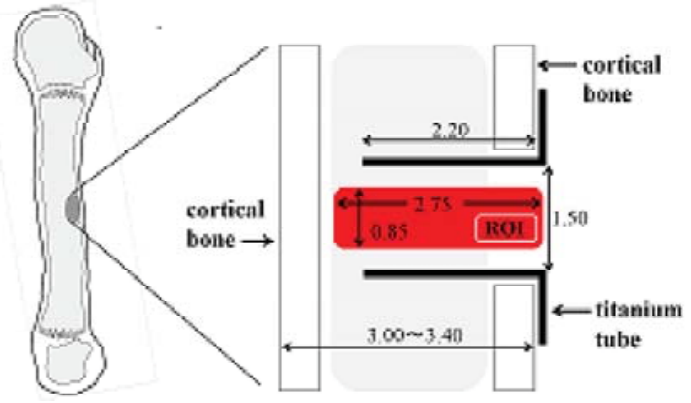

Figure 3. Schematic diagram of region of interest (ROI)

Tokyo, Japan) were used as senile osteoporosis model rats $(n=14)$. The rats were given food and water ad libitum and maintained on a 12-h light / dark cycle (lights on from 8:00 to 20:00) at $23 \pm 1{ }^{\circ} \mathrm{C}$ with $60 \pm 10 \%$ humidity during the experiment. The animal experiments in this study were conducted in accordance with the Tokyo Dental College Guidelines for Animal Experimentation (Approval number: 245101).

The rats were anesthetized by intraperitoneal injection ( 60 $\mathrm{mg} / \mathrm{kg}$ body weight) of sodium pentobarbital (Somnopentyl, Kyoritsu Seiyaku, Tokyo, Japan). The overlying skin of the femur was shaved and disinfected with iodated alcohol. The outside skin of the central femur was incised longitudinally, and the femur was exposed by stripping the periosteum. A bone defect (left femur, statin group; right femur, control group) was formed at the center of the femur using a round bar $1.5 \mathrm{~mm}$ in diameter under irrigation (Fig. 2a). The specimens were inserted into the bone defect using sterilized forceps. After implantation of the specimens, the muscle and skin were sutured (Fig. 2b).

All animals survived and recovered quickly from surgery. The rats appeared to be in good health throughout the test periods.

\section{Radiological assessment and measurement of newly formed bone}

New bone formation was quantitatively analyzed 7, 14, and 21 days after operation using a micro-computed tomography system $(\mu-\mathrm{CT})$. The defects in the femurs of the laboratory animals were scanned using $\mu$-CT (R-mCT ${ }^{\circledR}$, Rigaku, Tokyo, Japan) with 
Hiromitsu Yasuda et al.: Osteogenic Effect of Local Administration of Fluvastatin in Senile Osteoporosis Model Rats
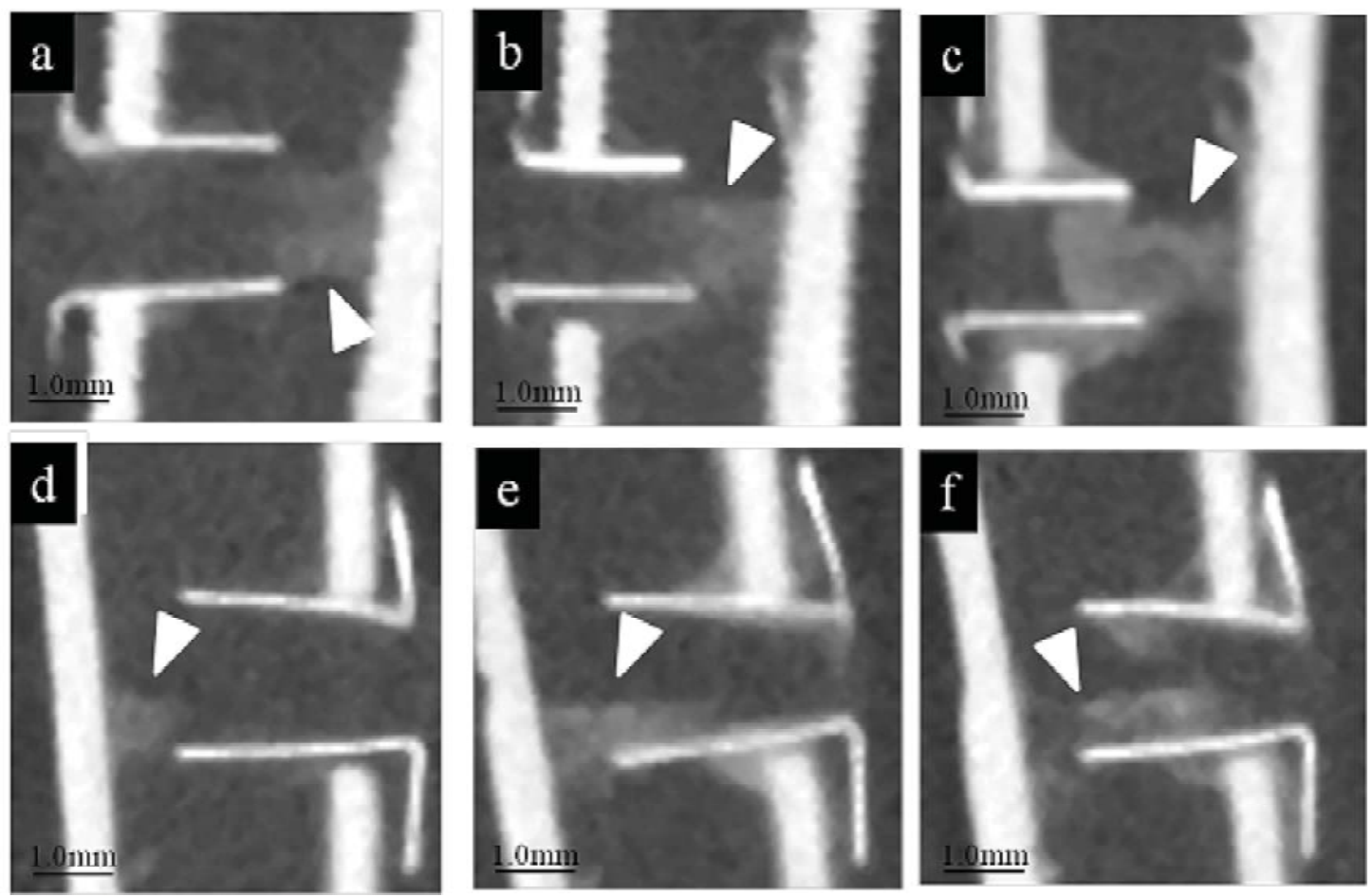

Figure 4. Representative $\mu$-CT images in the statin and control groups. Arrows indicate new bone formation. At 7 days, some slight new bone was seen to have formed in the statin group (a) and control group (d). At 14 days, obvious new bone was seen to have formed in the statin group (b) and control group (e). At 21 days, new bone was observed to have formed in the statin (c) and control (f) groups.

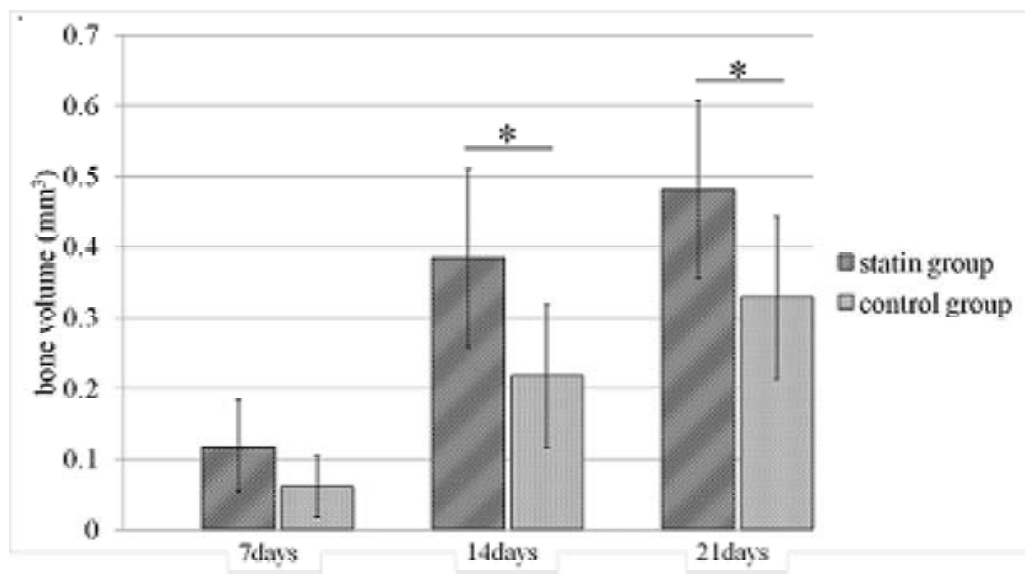

Figure 5. Bone volumes of newly formed bone in region of interest at days 7, 14, and 21.

an X-ray source of $90 \mathrm{kV} / 120 \mathrm{iA}$ at 7, 14, and 21 days after surgery $(n=8)$. After anesthesia with sodium pentobarbital, each rat was set on the object stage and imaging was performed over a full 360-degree rotation with an exposure time of $17 \mathrm{~s}$. An isotropic resolution of $50 \times 50 \times 50 \mu \mathrm{m}$ voxel size was selected in order to display the bone microstructure. Analysis of new bone formation was performed for each bone defect. TRI/3D-BON software (Ratoc System Engineering, Tokyo, Japan) was used to obtain 3dimensional (3-D) reconstructions from the obtained set of scans.
Of the entire 3-D data set, a cylindrical region of interest (ROI) with a diameter of $0.85 \mathrm{~mm}$ and height of $2.75 \mathrm{~mm}$ covering the entire thickness of the femur was selected for analysis (Fig. 3). Mask work was performed to binarize the 3-D data set in the ROI. Only 3-D images of new bone formation were displayed, and bone volumes were measured directly. All data were expressed as mean \pm standard deviation (SD). The Mann-Whitney U-test was used for statistical analysis. A $p$-value of less than 0.05 was considered statistically significant. 

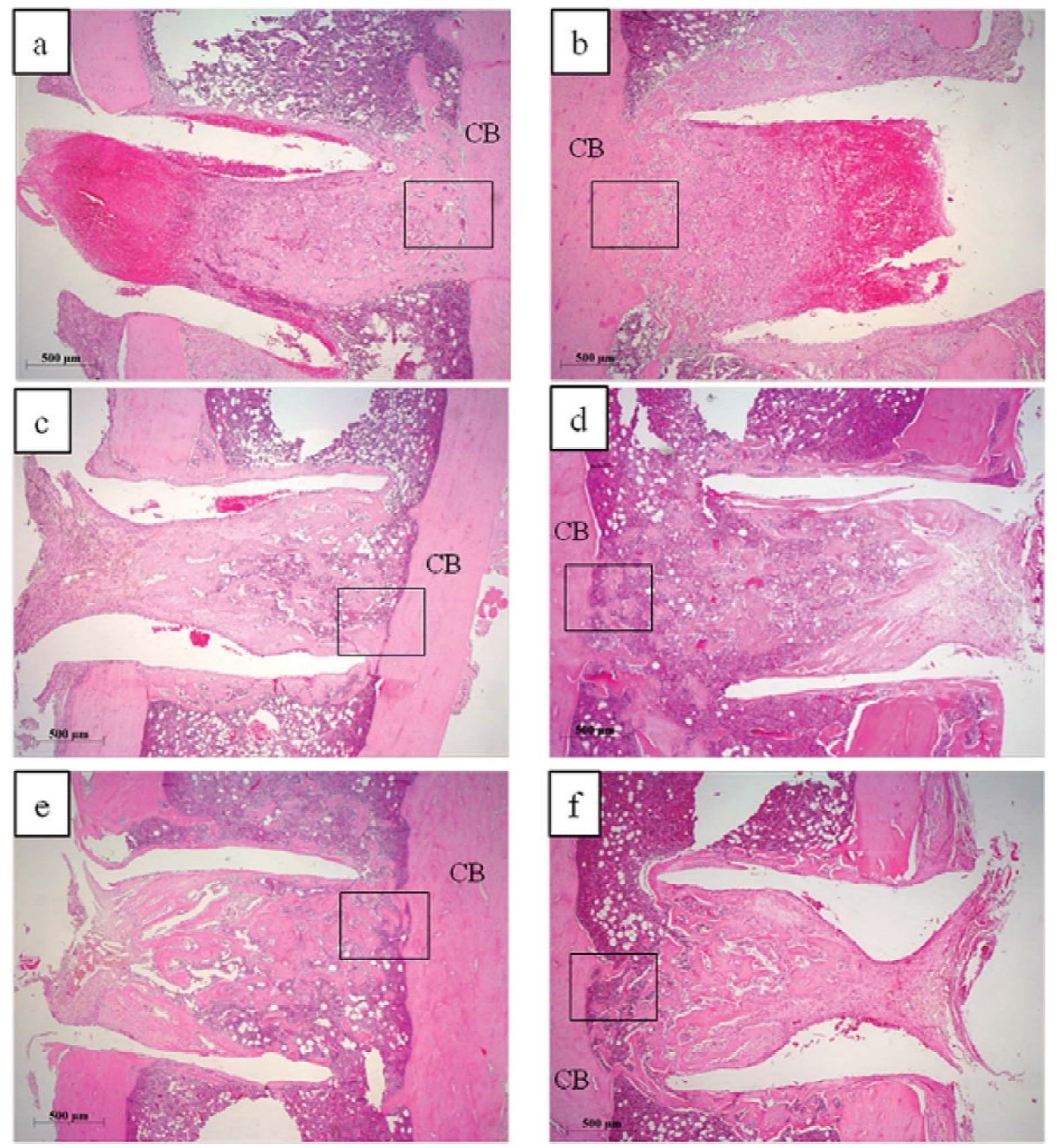

Figure 6. Representative histological analyses of newly formed bone in the statin (a, c, e) and control (b, d, f) groups. At day 7, hemorrhaging was recognized at the perforated region in both groups $(\mathrm{a}, \mathrm{b})$. At day 14, hemorrhaging at the perforated region had disappeared in both groups, and new bone was formed in the bone marrow space (c, d). At day 21, the volume of newly formed trabecular bone had increased in both groups compared to that at day $14(\mathrm{e}, \mathrm{f})$.

\section{Histologic examinations and immunohistochemical staining}

Animals were sacrificed at 7, 14, 21 days according to the following procedures. The femurs were separated with a diamond disk on a low-speed handpiece. The separated pieces were subjected to decalcification in a $10 \%$ neutral buffered formalin solution for 7 days. After decalcification, paraffin specimens were created with $10 \%$ EDTA (pH 7.0-7.5) (Wako Pure Chemical Industries, Osaka, Japan) for 6 days at room temperature. Then, the titanium tubes were carefully removed from the femurs. To observe the newly formed bone, the specimens were sliced into $3-\mu \mathrm{m}$ thick sections and stained with hematoxylin and eosin (H-E staining) according to standard protocols.

The samples were morphologically observed using a universal photomicroscope (Axiophot 2, Carl Zeiss, Oberkochen, Germany).

For immunohistochemical staining, the paraffin sections were deparaffinized with xylene and rehydrated using an ethanol series. The sections were washed in $10 \mathrm{nmol} / \mathrm{l}$ with $\mathrm{pH} 7.4$ phosphatebuffered saline (PBS) and endogenous peroxidase activity was 
Hiromitsu Yasuda et al.: Osteogenic Effect of Local Administration of Fluvastatin in Senile Osteoporosis Model Rats
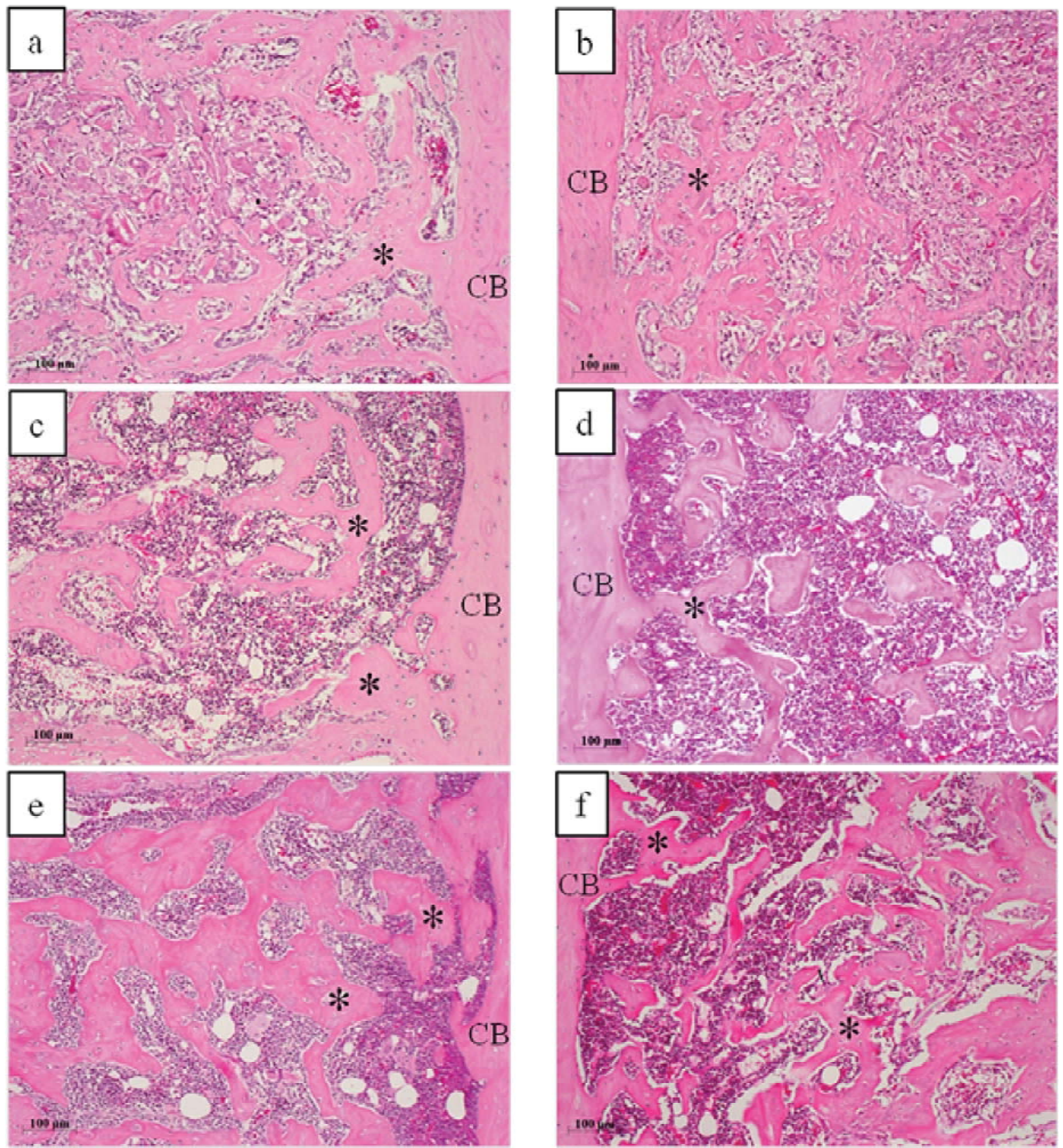

Figure 7. Histological analyses of newly formed bone corresponding to Fig. 6. (Images in Fig. 7a-f are higher magnification of areas shown in rectangle in Fig. 6a-f, respectively).

blocked by incubating sections with $0.3 \% \mathrm{H}_{2} \mathrm{O}_{2}$ in methanol for $30 \mathrm{~min}$. The sections were then reacted with the primary antibodies, rabbit anti-BMP-2 and Runx2 (Bioss Inc, MA, USA), diluted 1:100 overnight below $4{ }^{\circ} \mathrm{C}$. The sections were washed in PBS and then incubated with the secondary antibody, peroxidaselabeled anti-mouse IgG polyclonal antibody (Histofine Simple Stain Rat MAX-PO [MULTI]; Nichirei, Tokyo, Japan) for $30 \mathrm{~min}$ and washed with PBS. The sections were stained with 3,3'diaminobenzidine (DAB substrate kit, Nichirei, Tokyo, Japan), washed in sterilized water, and counterstained with hematoxylin. The sections were then dehydrated according to established protocol and the sections were examined and photographed using a universal photomicroscope (Axiophot 2).

\section{Results}

\section{Radiological analysis of newly formed bone}

In the $\mu$-CT images (Fig. 4, arrowheads), new bone formation increased with increased experimental periods. At 7 days after surgery, some slight new bone was seen to have formed in the bone marrow space of both the statin and control groups (Fig. 4a, $\mathrm{d}$ arrowheads). At 14 days after surgery, obvious new bone was seen to have formed in the bone marrow space of both the statin 

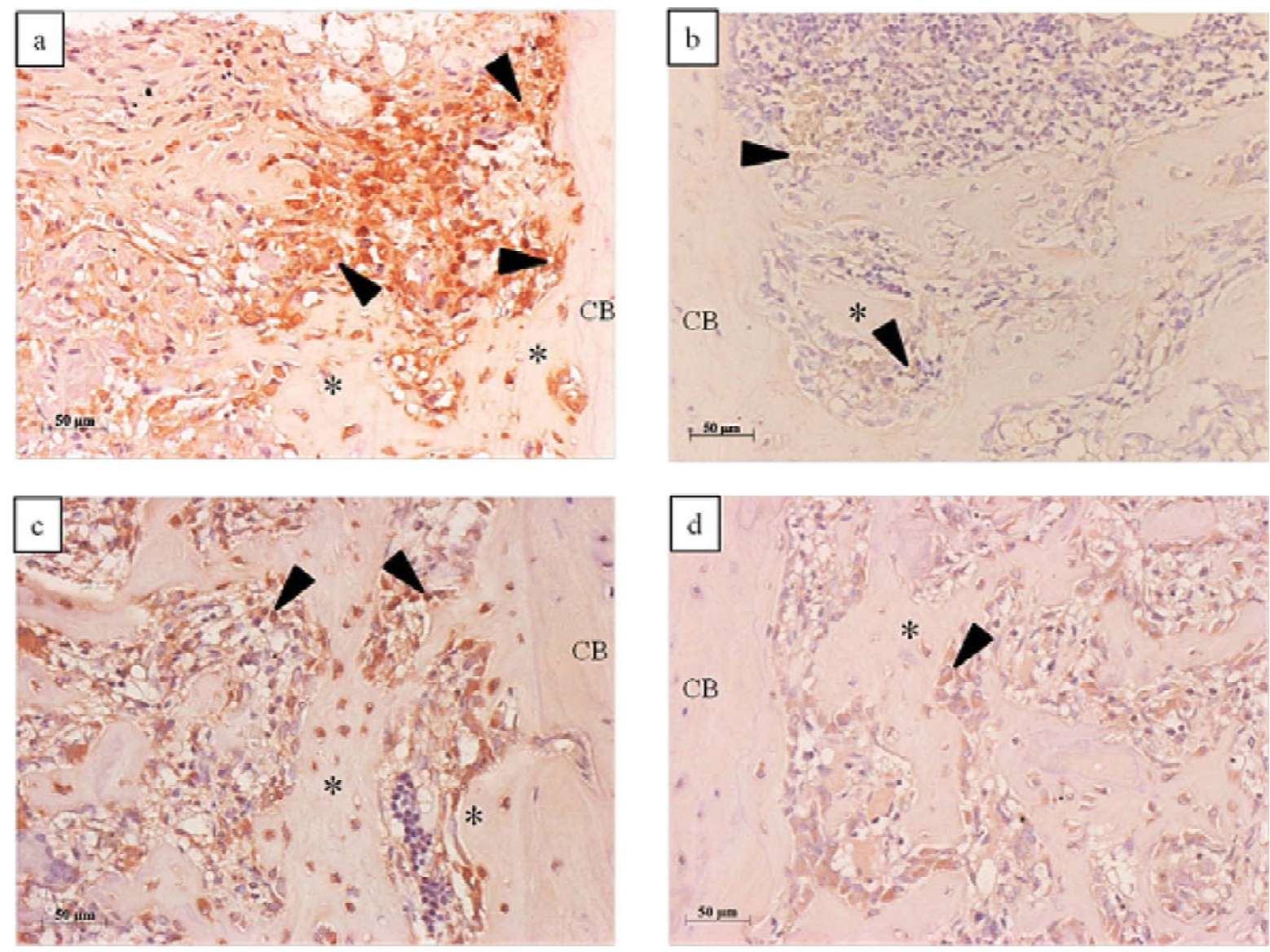

Figure 8. Immunohistochemistry of BMP-2 (a, b) and Runx2 (c, d) at day 7. A strong positive immune reaction with BMP-2 was seen in the statin group (a). In contrast, a weak positive immune reaction with BMP-2 was seen in the control group (b). The positive immune reaction with Runx 2 in the statin group was stronger than that in control group (c), despite the slight positive reaction with Runx2 that was seen in the control group (d).

and control groups. The newly formed bone was observed at the region close to the cortical bone that was on the side opposite that of the created bone defect (Fig. 4b, e arrowheads). At 21 days after surgery, new bone was observed to have formed in both groups (Fig. 4c, f arrowheads). At all time periods, higher levels of new bone were observed to have formed in the statin groups compared to those in the control groups.

\section{Bone volumes of newly formed bone}

Although the variations in the bone formation process between individuals were large, new bone formation was consistently observed in individual animals during the test periods. In addition, new bone formation in the statin groups was more advanced in each animal than in the control groups.

Fig. 5 showed the average bone volumes of the newly formed bone at days 7, 14, and 21. At day 7, no differences were found in bone volumes between the statin and control groups. At days 14 and 21 , the bone volumes of the newly formed bone in the statin group were significantly higher compared with those of the control group $(P<0.05)$.

\section{Histological assessment}

Figs. 6 and 7 showed the results of histological analyses of the newly formed bone. At day 7, hemorrhaging was recognized at the perforated region in both groups (Fig. 6a, b). New bone formation was observed at the region close to the cortical bone that was on the side opposite that of the created bone defect (Fig. $6 a, b$ and Fig. $7 a, b)$. Little difference was observed between the two groups.

At day 14, hemorrhaging at the perforated region had disappeared in both groups, and new bone was formed in the bone marrow space (Fig. 6c, d). The volume of newly formed trabecular bone had increased compared to that at day 7 . In addition, more new bone was seen to have formed in the statin group than in the control group (Fig. 6c, d and Fig. 7c, d).

At day 21, the volume of newly formed trabecular bone had increased in both groups compared to that at day 14. In the statin group, the bone marrow space was filled with new bone (Fig. 6e, f and Fig. 7e, f). 
Hiromitsu Yasuda et al.: Osteogenic Effect of Local Administration of Fluvastatin in Senile Osteoporosis Model Rats

\section{Immunohistochemical staining}

At day 7, a strong positive immune reaction with BMP-2 was seen in the osteoblasts localized on the surface of the newly formed bone and the outer periosteum around the marginal area of the existing bone in the statin group (arrowheads in Fig. 8a). In contrast, a weak positive immune reaction with BMP-2 was seen in the control group (Fig. 8b). The positive immune reaction with Runx2 in the statin group was stronger than that in control group (Fig. 8c), despite the slight positive reaction with Runx2 that was seen in the control group (Fig. 8d).

\section{Discussion}

In this study, we investigated the effect of the local administration of fluvastatin on bone formation in senile osteoporosis model rats. The results showed that new bone formation was enhanced by the local administration of fluvastatin by using a fluvastatin-gelatin complex. The positive reactions of BMP-2 and Runx2 were recognized in the statin group after 7 days.

Based on their characteristics, statins can be classified into hydrophobic or hydrophilic subgroups. Hydrophobic statins (simvastatin, lovastatin) enter the liver via the hepatic portal vein, while hydrophilic statins (rosuvastatin, pravastatin, fluvastatin) require active transport into the cell. ${ }^{23)}$ Simvastatin is administered by gavage and requires hepatic conversion to metabolically active â-hydroxy acid to become medicinally active. Therefore, for local administration, simvastatin would first have to be hydrolyzed to simvastatin acid ${ }^{24,25)}$.

In this experiment, fluvastatin was used to investigate the effect of the local administration of statins. The structure of fluvastatin is significantly different from those of previously used HMG-CoA reductase inhibitors such as the pravastatin and simvastatin metabolites, which are derived from fungi. ${ }^{26,27)}$ Therefore, it was considered that fluvastatin might have a pharmacological effect on bone formation in addition to the main effect of HMG-CoA reductase inhibitory activity. Some authors reported the positive effect of locally applied fluvastatin on the bone (Moriyama et al. 2008; Tanabe et al. 2011; Tanabe et al. 2012) $)^{22,28,29)}$. However, the mechanisms of the osteogenic effect of fluvastatin are not entirely clear.

In this study, a fluvastatin-gelatin complex was used as a carrier for fluvastatin $(300 \mu \mathrm{M})$ using $5 \mathrm{wt} \%$ gelatin hydrogel. Tanabe et al. reported that fluvastatin can be combined with gelatin as a complex. This combination is thought to be due to electrostatic coupling between the negatively charged fluvastatin and the positively charged alkaline gelatin. The fluvastatin-gelatin complex provides the same osteogenic activity as does fluvastatin used separately by HMG-CoA reductase. ${ }^{30)}$ It was reported that the fluvastatin $(1 \mathrm{mM})$-gelatin hydrogel delivery system promoted osteogenesis in rats ${ }^{22)}$. The results demonstrated that a specific amount of fluvastatin had the ability to bind to gelatin molecules, enabling local administration of fluvastatin in a drug delivery system and enhancing osteogenic activity. Accordingly, in this study, a fluvastatin-gelatin complex was used for the local administration of fluvastatin to investigate the facilitating effect on bone formation in senile osteoporosis model rats. In addition, in this study, a concentration of $300 \mathrm{iM}$ of fluvastatin was used for a preliminary experiment. The influence of the concentration of fluvastatin-gelatin complex on bone formation should be evaluated.

Osteoporosis is classified as postmenopausal osteoporosis, which involves an increase in bone resorption due to postmenopausal estrogen deficiency, and senile osteoporosis, which is reduced ossification associated with aging ${ }^{31}$. Postmenopausal osteoporosis is high-turnover osteoporosis resulting in trabecular bone reduction, while senile osteoporosis is low-turnover osteoporosis that affects both cortical and trabecular bone ${ }^{32)}$. Some researchers demonstrated bone formation using statins in ovariectomized rats ${ }^{14-17)}$. However, the ovariectomized rats were only used for the high-turnover osteoporosis model.

SHRSP rats were adopted as the senile osteoporosis model for experimental animals. Osteoporosis in SHRSP is known to start from age 3 months, and pathological conditions similar to low turnover, decreasing bone mass and amount of mineral content with aging, and a declining calcification and bone formation rate are seen ${ }^{33,34)}$. Thus, it was considered that the healing of bone in SHRSP would be slower than that in healthy animals or high turnover osteoporosis animals. Ogasawara et al. reported the effect of the oral administration of simvastatin using SHRSP for senile osteoporosis model rats ${ }^{13)}$. The 15 -weeks-old rats used in this study were considered highly suitable.

Several studies evaluated bone formation using $\mu-\mathrm{CT}^{13,22,35)}$. In this study, it was possible to observe the formation of new bone over time in the same individual animals using $\mu$-CT for laboratory animals $\left(\mathrm{R}-\mathrm{mCT}^{\mathbb{\circledR}}\right)$. In addition, this system enables the observation of new bone formation with a small sample because analysis is possible without sacrificing the animals at each radiographic time. Therefore, it is also beneficial from the viewpoint of animal welfare.

In the present study, new bone formation was observed by $\mu$ $\mathrm{CT}$ and histological evaluation in the region close to the cortical bone that was on the side opposite that of the created bone defect. In particular, the volumes of newly formed bone in the statin groups were significantly higher than were those of the control groups. Furthermore, in the results of immunohistochemical observation, positive reactions of BMP-2 and Runx 2 were observed in the statin group. Mundy et al. reported that statins could increase the expression of bone morphogenetic protein mRNA and, as a result, promoted bone formation ${ }^{5}$. These results suggested that the 
J.Hard Tissue Biology Vol. 23(4):389-398, 2014

enhanced expression of BMP-2 mRNA by fluvastatin was a trigger for osteoblast differentiation.

It was also observed that the bone marrow space of the statin group was filled with newly formed bone at day 21 , suggesting that the fluvastatin-gelatin complex was replaced by the newly formed bone ${ }^{22}$. Ayukawa et al. reported that local application of simvastatin enhanced bone formation in artificially created bone defects at an early stage. In addition, statins increased the expression of BMP-2 and ALP ${ }^{36)}$. Accordingly, favorable environments were created for bone formation by the local administration of fluvastatin.

Endosteal bone lining cells cover the majority of cortical bone surfaces $^{37-39)}$. Endosteal bone lining cells of the cortical bone were differentiated into cells that synthesized bone matrix by the local administration of a fluvastatin-gelatin complex. Therefore, new bone was actively formed from the region close to the cortical bone.

The local administration of fluvastatin differentiated the mesenchymal stem cells in the bone marrow and promoted the expression of BMP-2 from the endosteal bone lining cells.

In conclusion, The local administration of fluvastatin using a fluvastatin-gelatin complex facilitated bone formation in senile osteoporosis model rats.

\section{References}

1. Glösel B, Kuchler U, Watzek G and Gruber R. Review of dental implant rat research models simulating osteoporosis or diabetes. Int J Oral Maxillofac Implants 25: 516-524, 2010

2. Yang G, Song L, Guo C, Zhao S, Liu L and He F. Bone responses to simvastatin-loaded porous implant surfaces in an ovariectomized model. Int J Oral Maxillofac Implants 27: 369-374, 2012

3. Goldstein JL and Brown MS. Regulation of the mevalonate pathway. Nature 343: 425-430, 1990

4. Endo A. The discovery and development of HMG-CoA reductase inhibitors. J Lipid Res 33: 1569-1582, 1992

5. Mundy G, Garrett R, Harris S, Chan J, Chen D, Rossini G, Boyce B, Zhao M and Gutierrez G. Stimulation of bone formation in vitro and in rodents by statins. Science 286: 1946-1949, 1999

6. Song C, Guo Z, Ma Q, Chen Z, Liu Z, Jia H and Dang G. Simvastatin induces osteoblastic differentiation and inhibits adipocytic differentiation in mouse bone marrow stromal cells. Biochem Biophys Res Commun 308: 458-462, 2003

7. Junqueira JC, Mancini MN, Carvalho YR, Anbinder AL, Balducci I and Rocha RF. Effects of simvastatin on bone regeneration in the mandibles of ovariectomized rats and on blood cholesterol levels. J Oral Sci 44: 117-124, 2002

8. Skoglund B, Forslund C and Aspenberg P. Simvastatin improves fracture healing in mice. J Bone Miner Res 17: 2004-2008, 2002

9. Ayukawa Y, Okamura A and Koyano K. Simvastatin promotes osteogenesis around titanium implants. A histological and histometrical study in rats. Clin Oral Implants Res 15: 346350,2004

10. Saraf SK, Singh A, Garbyal RS and Singh V. Effect of simvastatin on fracture healing - an experimental study. Indian J Exp Biol 45: 444-449, 2007

11. Wang JW, Xu SW, Yang DS and Lv RK. Locally applied simvastatin promotes fracture healing in ovariectomized rat. Osteoporos Int 18: 1641-1650, 2007

12. Funk JL, Chen J, Downey KJ and Clark RA. Bone protective effect of simvastatin in experimental arthritis. J Rheumatol 35: 1083-1091, 2008

13. Ogasawara R, Furuya Y, Sasaki H, Yoshinari M and Yajima Y. Effect of oral administration of simvastatin on bone formation in senile osteoporosis rat. J Hard Tissue Biol 22: 459-469, 2013

14. Oxlund H and Andreassen TT. Simvastatin treatment partially prevents ovariectomy-induced bone loss while increasing cortical bone formation. Bone 34: 609-618, 2004

15. Du Z, Chen J, Yan F and Xiao Y. Effect of simvastatin on bone healing around titanium implant in osteoporosis rats. Clin Oral Implants Res 20: 145-150, 2009

16. Uyar Y, Baytur Y, Inceboz U, Demir BC, Gumuser G and Ozbilqin K. Comparative effects of risedronate, atorvastatin, estrogen and SERMs on bone mass and strength in ovariectomized rats. Maturitas 63: 261-267, 2009

17. Ho ML, Chen YH, Liao HJ, Chen CH, Hung SH, Lee MJ, Fu YC, Wang YH, Wang GJ and Chang JK. Simvastatin increases osteoblasts and osteogenic proteins in ovariectomized rats. Eur J Clin Invest 39: 296-303, 2009

18. Rogers MJ. Statins: lower lipids and better bones? Nat Med 6: 21-23, 2000

19. Yao W, Farmer R, Cooper R, Chmielewski PA, Tian XY, Setterberg RB, Jee WS and Lundy MW. Simvastatin did not prevent nor restore ovariectomy-induced bone loss in adult rats. J Musculoskelet Neuronal Interact 6: 277-283, 2006

20. Hodel C. Myopathy and rhabdomyolysis with lipid-lowering drugs. Toxicol Lett 128: 159-168, 2002

21. Guyton JR. Benefit versus risk in statin treatment. Am J Cardiol 97: 95C-97C, 2006

22. Tanabe K, Nomoto H, Okumori N, Miura T and Yoshinari M. Osteogenic effect of fluvastatin combined with biodegradable gelatin-hydrogel. Dent Mater J 31: 489-493, 2012

23. Tsartsalis AN, Dokos C, Kaiafa GD, Tsartsalis DN, Kattamis A, Hatzitolios AI and Savopoulos CG. Statins, bone formation and osteoporosis: hope or hype? Hormones 11: 
Hiromitsu Yasuda et al.: Osteogenic Effect of Local Administration of Fluvastatin in Senile Osteoporosis Model Rats

126-139, 2012

24. Yoshinari M, Hayakawa T, Matsuzaka K, Inoue T, Oda Y, Shimono M, Ide T and Tanaka T. Oxygen plasma surface modification enhances immobilization of simvastatin acid. Biomed Res 27: 29-36, 2006

25. Yoshinari M, Matsuzaka K, Hashimoto S, Ishihara K, Inoue T, Oda Y, Ide T and Tanaka T. Controlled release of simvastatin acid using cyclodextrin inclusion system. Dent Mater J 26: 451-456, 2007

26. Plosker GL and Wagstaff AJ. Fluvastatin: a review of its pharmacology and use in the management of hypercholesterolaemia. Drugs 51: 433-459, 1996

27. Hamelin BA and Turgeon J. Hydrophilicity / lipophilicity: relevance for the pharmacology and clinical effects of HMGCoA reductase inhibitors. Trends Pharmacol Sci 19: 26-37, 1998

28. Moriyama Y, Ayukawa Y, Ogino Y, Atsuta I and Koyano K. Topical application of statin affects bone healing around implants. Clin Oral Implants Res 19: 600-605, 2008

29. Tanabe K, Saima H, Suzuki K, Miura T and Yoshinari M. Effect of fluvastatin release on local osteogenicity in rat calvaria. J Oral Tissue Engin 8: 181-187, 2011

30. Tanabe K, Miura T and Yoshinari M. Electrostatically coupled state of fluvastatin with gelatin in vivo. J Hard Tissue Biol 22: 449-452, 2013

31. Riggs BL, Khosla S and Melton LJ3rd. A unitary model for involutional osteoporosis: estrogen deficiency causes both type I and type II osteoporosis in postmenopausal women and contributes to bone loss in aging men. J Bone Miner Res 13: 763-773, 1998

32. Duque G and Troen BR. Understanding the mechanisms of senile osteoporosis: new facts for a major geriatric syndrome. J Am Geriatr Soc 56: 935-941, 2008

33. Yamori Y, Fukuda S, Tsuchikura S, Ikeda K, Nara Y and Horie R. Stroke-prone SHR (SHRSP) as a model for osteoporosis. Clin Exp Hypertens A 13: 755-762, 1991

34. Fukuda S and Yamori Y. Characteristics of bone metabolism in the spontaneously hypertensive rat as a model of spontaneously developing osteoporosis. In: Handbook of Bone Morphometry Second edition, ed. by Takahashi H, Nishimura Shoten, Niigata, 1997, pp 149-158

35. Yoshida A, Sasaki H, Furuya Y, Yoshinari M and Yajima Y. Effect of low-intensity pulsed ultrasound on bone-healing process in murine low-turnover osteoporosis model. J Hard Tissue Biol 22: 301-310, 2013

36. Ayukawa Y, Yasukawa E, Moriyama Y, Ogino Y, Wada H, Atsuta I and Koyano K. Local application of statin promotes bone repair through the suppression of osteoclasts and the enhancement of osteoblasts at bone-healing sites in rats. Oral Surg Oral Med Oral Pathol Oral Radiol Endod 107: 336-342, 2009

37. Luk SC, Nopajaroonsri C and Simon GT. The ultrastructure of endosteum: a topographic study in young adult rabbits. J Ultrastruct Res 46: 165-183, 1974

38. Matthews JL, Wiel CV and Talmage RV. Bone lining cells and the bone fluid compartment, an ultrastructural study. Adv Exp Med Biol 103: 451-458, 1978

39. Miller SC, Bowman BM, Smith JM and Jee WS. Characterization of endosteal bone-lining cells from fatty marrow bone sites in adult beagles. Anat Rec 198: 163-173, 1980 
J.Hard Tissue Biology Vol. 23(4):389-398, 2014 